\title{
Determinación de la digestibilidad y degradabilidad cecal y total de materias primas como: guaratara (Axonopus purpusii) y hojas de plátano (Musa sp) en chigüiros (Hydrochoerus hydrochaeris) en cautiverio y su relación con indicadores metabólicos
}

\section{Determination of cecal and total digestibility and degradability of raw materials as guaratara (Axonopus purpusii) banana leaft (Muse sp) in chigüiros (Hydrochoerus hydrochaeris) in captivity and their relationship with metabolic indicators}

Ochoa Miguel H. ${ }^{1}$, Céspedes Olinda Y. ${ }^{1}$ y Fuentes Edgar E. ${ }^{2}$ ${ }^{1}$ Medicos Veterinarios Zootecnistas, ${ }^{2} \mathrm{MVZ}$. MSc. PhD Docente Unillanos efuentes@unillanos.edu.co

Recibido 14 de Febrero 2012, Aprobado 18 de Abril 2012

\section{RESUMEN}

El propósito de la investigación fue determinar la digestibilidad y degradabilidad cecal y total en chigüiros (Hydrochoerus hydrochaeris) en cautiverio, lo cual se realizó en el municipio dé Paz de Ariporo, vereda Normandía, finca la Providencia ubicada a cuatro (4) horas del casco urbano en el departamento del Casanare; con el fin de determinar la composición nutricional del pasto guaratara (Axonopus purpusii) y hojas de plátano (Musa sp.), como base de la alimentación de chigüiros en cautiverio. Se utilizaron cuatro (4) animales clínicamente sanos, de peso promedio entre $30 \mathrm{~kg}$ y $35 \mathrm{~kg}$ con un diseño experimental en cuadrado latino, conformados por parejas y sometidos a etapas de acostumbramiento - adaptación de ocho (8) días, periodo en el cual se suministró guaratara (Axonopus purpusii) y hojas de plátano (Musa sp.) y agua diariamente a voluntad. Se realizó el proceso de digestibilidad cecal y total con bolsas móviles. Las dos fuentes alimenticias fueron previamente sometidas a secado y posteriormente molido para ser incorporados en las bolsas móviles y recolectado en la materia fecal, para ser enviado al laboratorio, con el fin de realizar el análisis bromatológico (FDA, FDN, 
PC, grasa, carbohidratos, y cenizas). Para determinar la digestibilidad total, las bolsas fueron introducidas mediante sonda oro gástrica y la digestibilidad cecal se determinó por fistula cecal (cánula cecal y cánula de PVC en ciego). En cada uno de los ensayos se hizo recolección de suero sanguíneo para hacer la correlación de perfiles metabólicos con el grado de digestibilidad. Se tomaron muestras de suelo para su respectivo análisis. Se concluye que la hoja de plátano es altamente palatable al igual que el guaratara. Además, la metodología fue pertinente para la recolección de los datos.

Palabras claves: Chigüiro, guaratara, hoja de plátano, digestibilidad, perfil metabólico.

\section{ABSTRACT}

The purpose of the research was to determine the digestibility and degradability and total cecal in chigüiro (Hydrochoerus hydrochaeris) in captivity, which was held in the municipality of Paz de Ariporo, Normandía village, Providencia farm located four (4) hours of the hull city in the department of Casanare, in order to determine the nutritional composition of guaratara grass (Axonopus purpusii) and leaves of banana (Musa sp.) as a staple food of capybaras in captivity. We used four (4) clinically healthy, average weight between $30 \mathrm{~kg}$ and $35 \mathrm{~kg}$ with a Latin square experimental design, made in pairs and subjected to stages of adaptation adaptation of eight (8) days, a period in which provided guaratara (Axonopus purpusii) and leaves of banana (Musa sp.) and water ad libitum daily. Process was performed cecal digestibility and total mobile bags. The two food sources were previously subjected to drying and then grinding to be incorporated into mobile bags and collected in the stool, to be sent to the laboratory to perform chemical composition analysis (ADF, NDF, CP, fat, carbohydrates, and ash). To determine the total digestibility, bags were introduced by gold gastric tube and cecal digestibility was determined by cecal fistula (cecal cannula and cannula PVC blind). In each test was collected for serum metabolic profiles correlation with the 
degree of digestibility. Soil samples were taken for examination. We conclude that the banana leaf is highly palatable as well as the guaratara. Furthermore, the methodology was appropriate for data collection.

Keywords: Chigüiro, guaratara, banana leaf, digestibility, metabolic profile.

\section{INTRODUCCIÓN}

El chigüiro (Hydrochoerus hydrochaeris) en cautiverio y su relación con indicadores metabólicos, es de vital importancia dado que brinda la oportunidad de acceder al perfeccionamiento de las competencias relacionadas con la generación de nuevo conocimiento y la profundización en las diferentes áreas del saber, esto enfocado a sistemas de producción en especies silvestres promisorias como lo es el chigüiro que busca percibir opciones de producción con uso racional de los recursos naturales, otorgando alternativas para la generación de paquetes tecnológicos con aplicabilidad en la zoocría, siendo este un renglón importante en el desarrollo científico y económico de la Orinoquia Colombiana. Dentro de la búsqueda para el desarrollo de este tipo de procesos, la nutrición es la base fundamental que garantiza el éxito productivo, mediante la evaluación de alimentos propios de la región y algunos otros de fácil establecimiento, definiendo así mismo, dietas balanceadas que garanticen el desarrollo óptimo de la especie y de su actividad reproductiva; haciendo de este modo rentable y sostenible la producción de carne, sin afectar las poblaciones naturales de la especie.

Los chigüiros requieren sitios secos para descansar y alimentarse y cuerpos de agua para bañarse, beber, copular y refugiarse de algunos depredadores (Ojasti y Burgos, 1985). Se encuentran asociados a varios tipos de hábitats cercanos a cuerpos de agua, como selvas húmedas, bosques secos, matorrales, y sabanas (Emmons, 1997). Las mayores densidades de chigüiros se encuentran en la zona pantanosa de Mato Grosso brasilero y en las sabanas de Colombia y Venezuela (FAO, 1985). 
Los estudios ecológicos en ambientes naturales son necesarios para entender los requerimientos de diferentes especies y así poder generar modelos de uso sostenible, para especies útiles, como el chigüiro. Las especies forrajeras Axonopus purpusii, Andropogon bicornis y Panicum sp., cubren el $52,9 \%$ ( $\pm 5,2$ ES) y $55,3 \%$ ( $\pm 5,3$ ES) en la época lluviosa y seca, respectivamente. La guaratara (Axonopus purpisii) es la especie con mayor aporte a la dieta a lo largo de las dos épocas y su consumo se hace principalmente en los bancos. Las plantas de estero presentan un incremento en su consumo durante la estación seca. Las especies con mayor nivel de preferencia son plantas típicas de bajo y estero, como Murdania nudiflora y Eleocharis mínima; El chigüiro puede estar presentando un cambio en su tipo de alimentación según la época, de cualitativa en el invierno, a alimentación balanceada en el verano como adaptación a la marcada estacionalidad llanera. La alta proporción de pasto guaratara en la dieta la sugiere como una especie importante para la sobrevivencia de este roedor, más que plantas con un alto grado de preferencia. Las principales plantas consumidas pertenecen a las familias Poaceae, Cyperaceae, Pontederiaceae, Fabaceae, Mimosaceae y Caesalpinaceae (Forero et al., 2003).

Los chigüiros empleados en el proyecto fueron capturados en su habitad natural en los predios de la finca la Providencia, en donde las pasturas nativas predominantes son: guaratara (Axonopus purpusii), rabo de zorro (Andropogon bicornis), grama (Paspalum contractum), lambedora (Leersia hexandra) y paja peluda (Trachypogon vestitus).

\section{MATERIALES Y MÉTODOS}

Este trabajo de investigación se realizó en el departamento de Casanare, municipio de Paz de Ariporo, vereda Normandía, finca la Providencia. El proceso de captura se realizó en horas de la tarde con la colaboración de personas de la región (Figura 1), quienes brindaron su apoyo motivados por el proceso investigativo. Los corrales fueron construidos con guadua y postes de madera 
(Figura 2), con unas dimensiones de $4 \times 4$ metros y 2 metros de altura, cada uno de estos dotado de un pozo artificial (Figura 3 ).

Cuatro (4) animales hembras fueron sometidos a 8 días de acostumbramiento con una dieta a base de guaratara (Axonopus purpusii) (Tabla 1), hoja de plátano (Musa sp.) y agua a voluntad. El manejo diario consistía en el cambio periódico de agua, recolección de heces y restos de alimento.

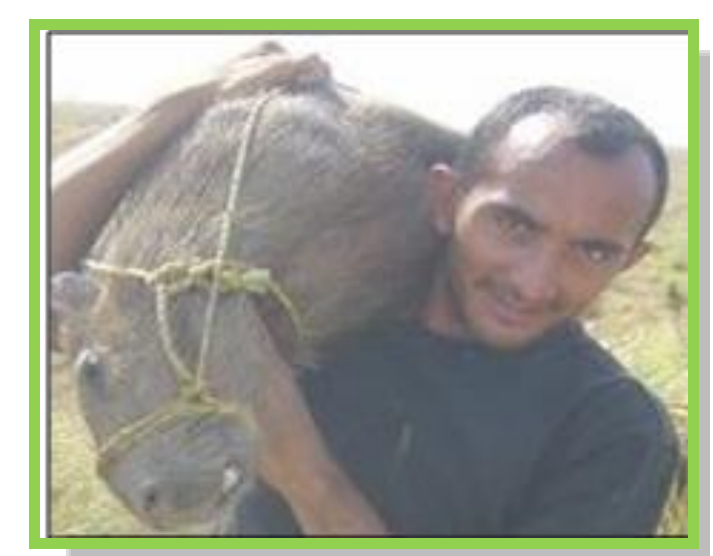

Figura 1. Captura de chigüiros

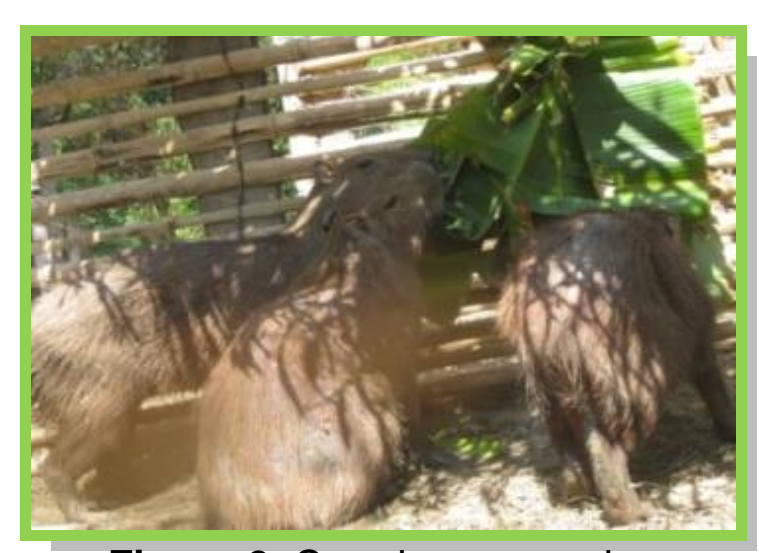

Figura 2. Corrales en guadua

Tabla 1. Composición bromatológica del guaratara (Axonopus purpusii)

\begin{tabular}{ccccccccccc}
\hline $\begin{array}{c}\text { Estación } \\
\text { climática }\end{array}$ & $\begin{array}{c}\text { DIVMS } \\
\%\end{array}$ & $\begin{array}{c}\mathbf{P C} \\
\%\end{array}$ & $\begin{array}{c}\mathbf{C a} \\
\%\end{array}$ & $\begin{array}{c}\mathbf{P} \\
\%\end{array}$ & $\begin{array}{c}\mathbf{K} \\
\%\end{array}$ & $\begin{array}{c}\mathbf{M g} \\
\%\end{array}$ & $\begin{array}{c}\mathbf{C u} \\
\%\end{array}$ & $\begin{array}{c}\mathbf{M n} \\
\mathbf{p p m}\end{array}$ & $\begin{array}{c}\mathbf{Z n} \\
\mathbf{p p m}\end{array}$ & $\begin{array}{c}\mathbf{F e} \\
\mathbf{P p m}\end{array}$ \\
\hline Lluvia & 54.8 & 7.3 & 0,17 & 0.11 & 0.92 & 0.14 & 6.4 & 101 & 47 & 253 \\
Transición & 54.9 & 6.5 & 0.31 & 0.11 & 0.67 & 0.22 & 4 & 115 & 27 & 235 \\
Seca & 51.5 & 5.9 & 0,21 & 0.08 & 0.48 & 0.14 & 3 & 123 & 40 & 288 \\
Media & 54.7 & 6.4 & 0.23 & 0.10 & 0.68 & 0.17 & 4 & 133 & 38 & 259 \\
\hline
\end{tabular}

Fuente: Forero et al., (2003).

Paralelo al manejo de los animales en periodo de acostumbramiento, se realizó recolección del alimento, guaratara (Axonopus purpusii) y hoja de plátano (Musa 
$s p)$; para este procedimiento fue importante la búsqueda de bancos representativos en donde aún pese al intenso verano se conservarán en estado fresco. El secado (Figura 4) se realizó al sol en láminas de zinc y en dado caso con calor en estufa. El molido se realizó en molino manual, y dado a esto se vio la necesidad de tamizar las muestras para lograr homogeneidad y además eliminar partículas muy pequeñas que pudiesen atravesar fácilmente por la porosidad de la tela de nylon utilizada en las bolsas móviles. Se determinó del porcentaje de materia seca de guaratara (Axonopus purpusii) y hoja de plátano (Musa sp) (Tabla 2).

Se elaboraron bolsas en tela (Figura 5) de "tul" ( $0.5 \mathrm{~cm}$ de ancho $\times 3 \mathrm{~cm}$ largo), las cuales fueron fina y detalladamente terminadas dado a que estas se encontrarían en contacto directo con la mucosa esofágica y gastrointestinal y un material abrasivo generaría graves daños en la integridad anatomo-fisiológica de la misma. Las muestras de guaratara (Axonopus purpusii) y hoja de plátano (Musa sp) se pesaron en balanza electrónica (Figura 6.), estandarizando pesos aproximados de $0.60 \mathrm{~g}$ por bolsa móvil. Para fines de identificación de las mismas se utilizaron hilos de colores (azul, gris y blanco = harina de hoja de plátano y negro verde y rosado = harina de guaratara).

Tabla 2. Determinación de materia seca en guaratara (Axonopus purpusii) y hoja de plátano (Musa sp.)

Guaratara (Axonopus purpusii) Hoja de plátano (Musa sp.)

\begin{tabular}{cccccc}
\hline $\begin{array}{c}\text { Peso fresco } \\
(\mathbf{g})\end{array}$ & Peso Seco (g) & \% M.S. & $\begin{array}{c}\text { Peso fresco } \\
\text { (gr) }\end{array}$ & $\begin{array}{c}\text { Peso seco } \\
\text { (gr) }\end{array}$ & \% M.S. \\
\hline 50.00 & 30.98 & 61.96 & 50.04 & 2.68 & 5.34 \\
\hline
\end{tabular}

Posterior al periodo de acostumbramiento, el día 9 se procedió al inicio de la fase experimental con la introducción de las muestras, para lo cual fue necesario tranquilizar y anestesiar a cada uno de los animales: se aplicó el protocolo 
anestésico consistente en xilacina $0.2 \mathrm{mg} / \mathrm{kg}$ IV y diazepam $0.1 \mathrm{mg} / \mathrm{kg}$ IV (vena cefálica).

Se realizo toma de muestra de sangre por medio de venipuncion (Figura 7 ) en la vena femoral (utilizando agujas hipodérmicas calibre 21 conectadas a tubos vacutainer de $10 \mathrm{ml}$ ). Ésta se utilizó para medición de glicemia (Figura 8) y obtención de suero sanguíneo, para este último la sangre fue centrifugada a 3.000 r.p.m. por 15 minutos, el suero se almaceno en tubos de transporte y fueron debidamente refrigerados.

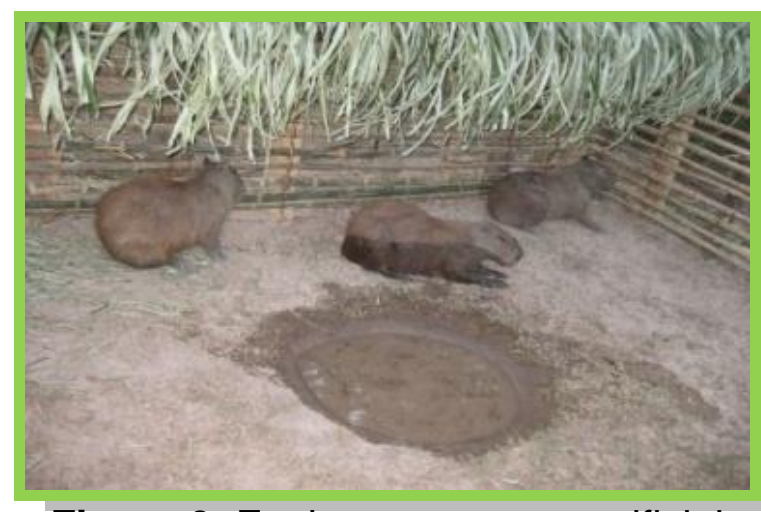

Figura 3. Encierro con pozo artificial

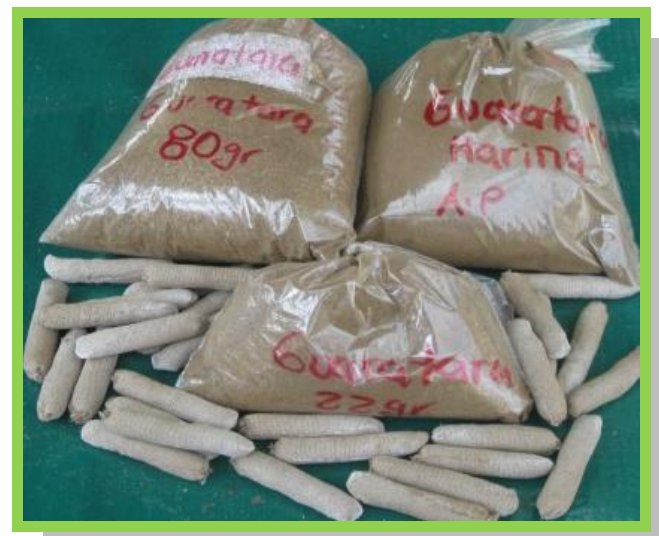

Figura 5. Bolsas en tela

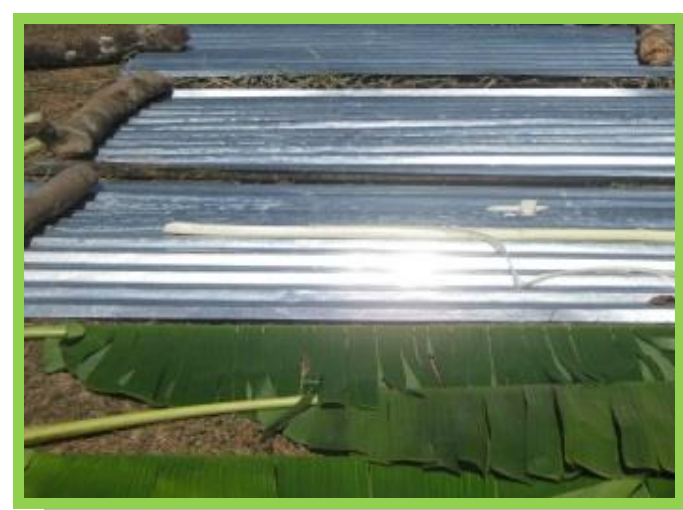

Figura 4. Secado en láminas de zinc

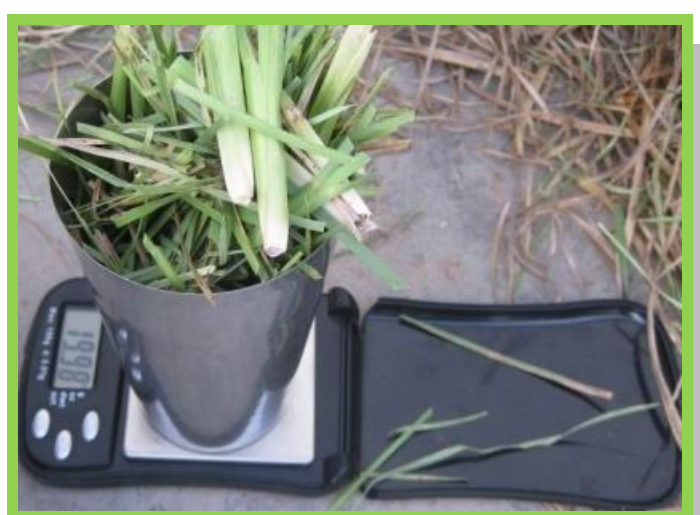

Figura 6. Pesaje en balanza electrónica

Se colocó el animal en decúbito lateral izquierdo, instaurando la restricción adecuada a nivel de los incisivos superiores e inferiores. La sonda orogástrica fue 
lubricada interna y externamente (con aceite de cocina) a fin de permitir el correcto desplazamiento a través del esófago y el deslizamiento rápido y efectivo de las bolsas móviles al interior de la misma. Se introdujo un tubo protector previamente adaptado para salvaguardar la sonda a nivel de la entrada de la cavidad bucal, luego se colocó la sonda (Figura 9) con especial cuidado para evitar laceraciones de la mucosa esofágica y además teniendo en cuenta que la ubicación de la misma fuese a una distancia prudente a nivel de la curvatura cervical que es un tanto prominente.

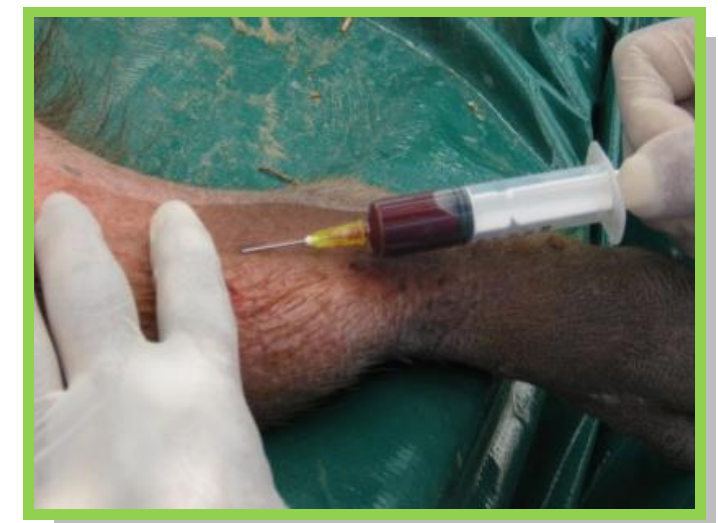

Figura 7. Venipunción en vena femoral

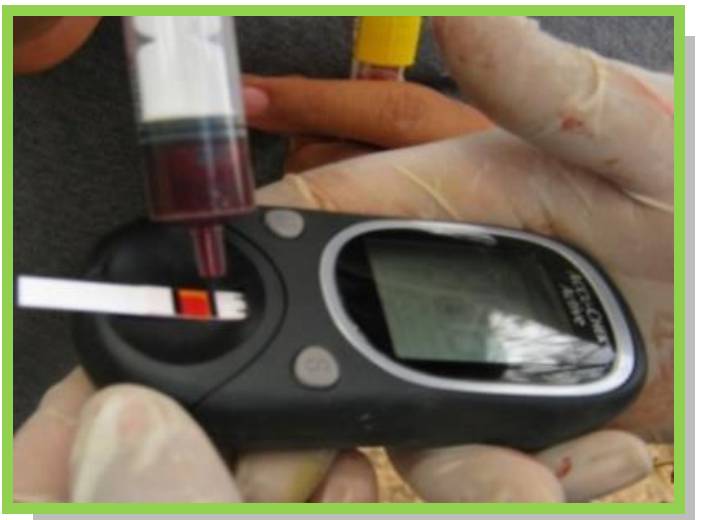

Figura 8. Medición de glicemia

Posteriormente los animales fueron colocados en el corral correspondiente el cual no contaba con disposición de agua hasta que el animal se recuperara a cabalidad del estado anestésico para la introducción de las bolsas, el paso siguiente consistió en la revisión periódica (cada 1 o 2 horas) de cada uno de los corrales donde se encontraban los animales experimentales a fin de retirar las heces y por ende recuperar las muestras contenidas en las bolsas móviles, llevando un registro claro de la hora de recolección junto con el número de bolsa móvil. Las bolsas se lavaron el número de veces necesarias hasta que el agua quedo limpia, posteriormente se colocaron al sol y al calor en estufa.

Luego de haber realizado el proceso experimental de digestibilidad y degradabilidad total, se destinó uno de los especímenes experimentales para 
llevar a cabo la segunda fase del proyecto consistente en la determinación de digestibilidad y degradabilidad cecal. Para ello el animal fue sometido a un proceso quirúrgico usando la técnica descrita por Drogoul et al., (2000), en el ijar derecho (Figura 10) para colocar la fístula cecal (cánula) (Figura 11) de cloruro de polivinilo (PVC) en el ciego. Se realizaron 6 repeticiones, y se determinó para la base alimenticia guaratara (Axonopus purpusii) a las 6 y 18 horas. Para esto fue necesario elaborar bolsas de nylon de $2 \mathrm{~cm}$ de ancho por $6 \mathrm{~cm}$ de largo en donde se empacaron $7 \mathrm{gr}$ de la muestra y fueron sujetadas a un nylon para facilitar la extracción de las mismas (Figura 12).

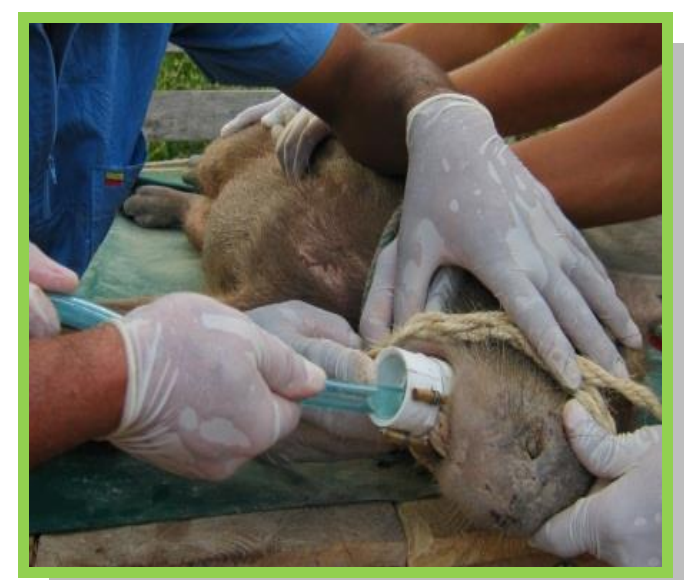

Figura 9. Paso de sonda orogástrica

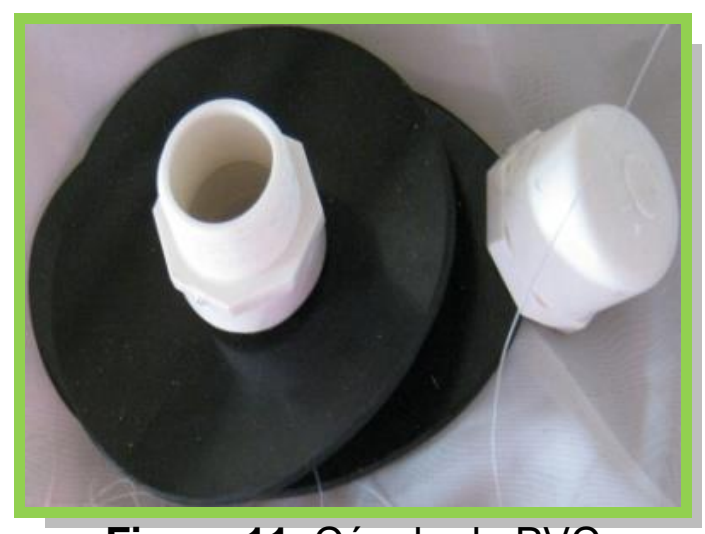

Figura 11. Cánula de PVC

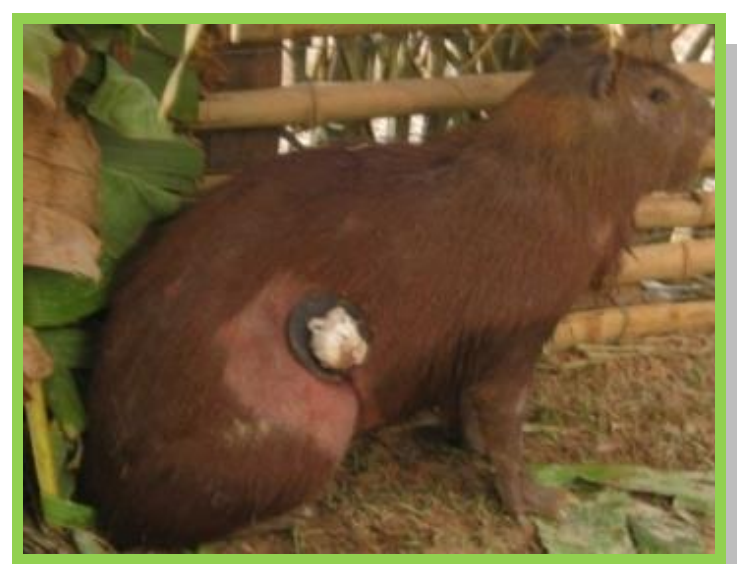

Figura 10. Fistulación en ijar derecho

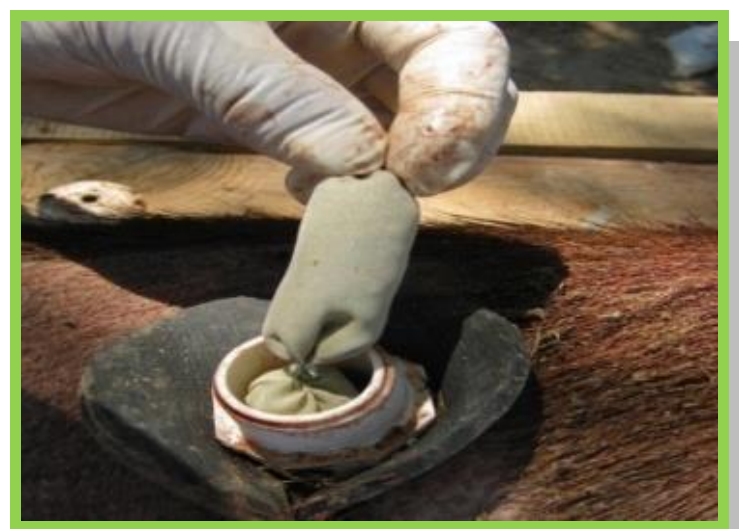

Figura 12. Introducción de bolsas en el ciego 
Luego de ser retiradas las muestras a las 6 y 18 horas respectivamente, se realizó el mismo proceso de lavado y secado que las muestras de digestibilidad y degradabilidad total. Las muestras de las dos fases experimentales fueron pesadas, clasificadas, y empacadas en recipientes plásticos (frascos de muestra de orina) para evitar contaminación o perdidas de muestra.

Las muestras de suelo fueron recolectadas de 3 puntos claves dentro del área en donde se encontraba establecido el guaratara (Axonopus purpusii) y el plátano (Musa $s p$ ), las muestras de estos 3 puntos fueron homogenizadas y de esta forma se tomaron $500 \mathrm{~g}$ para ser enviados al laboratorio de suelos de la Universidad de los Llanos para el respectivo análisis.

\section{RESULTADOS Y DISCUSIÓN}

La digestibilidad varía de 47.8 a 66.0 , de 32.7 a 48.8 , de 36.0 a 56.7 , de 41,1 a 66.9, y de 52.4 a $64.6 \%$ en $A$. purpusii, L. lanatum, P. laxum, L. hexandra, e $H$. amplexicaulis, respectivamente. Los valores medios alcanzan a 39.2, 44.7, 50.6, 53.7 y $58.7 \%$ en $L$. lanatum, $P$. laxum, L. hexandra, A. purpusii e $H$. amplexicaulis respectivamente. En sabanas altas, durante la época lluviosa, la DISMS fluctúa de 47.8 a $66.0 \%$ en $A$. purpusii y de 32.7 a $48.8 \%$ en $L$. lanatum. La media general desciende de 54.8 a $51.5 \%$ y de 39.8 a $39.1 \%$ en las mismas especies durante las épocas lluviosa y seca, respectivamente. Los valores más elevados encontrados en $A$. purpusii se explican posiblemente porque la relación hoja-tallo y el índice de área foliar del estrato superior de esta especie superan a la encontrada en $L$. lanatum (Tejos, 1994). Los valores de DISMS se encuentran dentro de los rangos señalados por la literatura (González et al., 1981; Muñoz et al., 1986a,b; Braga y Camarao, 1987; Nascimento et al., 1990). Desde un punto de vista de producción animal es deseable que las especies forrajeras tengan valores elevados de DISMS y para el caso de $A$. purpusii podría ser consumida durante todo el año, pero en la época lluviosa se obtienen digestibilidades más elevadas. En cambio, en $L$. lanatum la DISMS fue baja durante todo el año, con la excepción del rebrote a las 
cuatro semanas. Desde un punto de vista de utilización de esta última especie forrajera pareciera conveniente planificar intervalos de pastoreos entre 4 a 6 semanas porque períodos de descanso más largos causan una disminución substancial de la digestibilidad. En $A$. purpusii, a pesar que presenta valores medios de DISMS, sería conveniente también planificar intervalos entre pastoreos que no excedieran las seis semanas durante la época lluviosa. En sabanas de bajío, antes de la inundación del área, la digestibilidad fluctúa de 47.8 a $54.8 \%$ en $P$. laxum y de 59.0 a $60.4 \%$ en $L$. hexandra. Una vez desaparecida la inundación, la DISMS de estas especies varía de 36.0 a $54.2 \%$ y de 46.2 a $50.6 \%$, respectivamente. Estos valores son similares a los señalados por González et al., (1981) y Tejos y Arias, (1988). De las dos especies de bajíos estudiadas, $L$. hexandra, sostenidamente, presenta valores de DISMS más elevada a lo largo del año y es al inicio de la época lluviosa cuando se alcanzan los valores máximos. En sabanas altas, durante el período de utilización preferente los contenidos proteicos fluctúan de 5.3 a 10.1 y de 4.8 a $8.9 \%$ en $A$. purpusii y L. lanatum, respectivamente, y coinciden con resultados obtenidos en gramíneas nativas tropicales por Muñoz et al., (1986a,b), Tejos, (1986) y Morales y Berroterán, (1991). A las cuatro semanas del corte de uniformidad los valores proteicos se incrementan y sobrepasan el nivel crítico del 7\% señalado por Minson, (1981) y después de ese momento el contenido proteico desciende semanalmente de 0.25 a $0.58 \%$.

En la composición química proximal de la harina de plátano se encontró un contenido proteico inferior al observado por Van Severen y Carbonell, (1949). Igualmente se encontró un desequilibrio en la proporción calcio/fósforo, tal como ya se ha mencionado.

En cuanto al tiempo de transito de las bolsas móviles se evidencia un intervalo de tiempo entre las 9 horas y las 115 horas, es un rango disperso en donde el mayor porcentaje de excreción de bolsas se da a las 20 horas (15-20 horas específicamente) con un $17 \%$ esto se interpretaría como un dato normal 
correlacionado con que se lleva a cabo de forma natural. En cuanto al 5 y $3 \%$, a las 110 y 115 horas respectivamente, éste corresponde a uno de los animales experimentales que presentó sintomatología respiratoria (estertores, secreción nasal bilateral escasa) a los 8 días post captura, esto conllevó a la disminución del consumo de alimento (hipomotilidad) y por ende aumento del tiempo de tránsito intestinal presentándose un tiempo prolongado de excreción de las bolsas móviles.

El porcentaje de pérdida de las bolsas se explica desde dos puntos de vista básicamente, en primera instancia durante la recolección de las heces se evidenciaron fibras pertenecientes al material de las bolsas móviles, lo cual indica que algunas de las muestras fueron regurgitadas, molidas y tragadas; por otro lado es factible que el porcentaje restante de perdida a pesar de la constante supervisión se haya dado por perdida accidental dentro del área del corral dado a pisoteo por parte de los animales.

\section{CONCLUSIONES}

Los estudios realizados son de gran importancia porque permiten hacer uso sostenible, conservación y preservación de especies silvestres como lo es el chigüiro (Hydrochaerus hydrochaeris).

La investigación y la difusión de los resultados de las actividades de manejo que se realizaron durante la fase experimental son de gran importancia para futuras investigaciones en esta especie.

Es necesario reforzar la investigación aplicada a esta especie debido a la necesidad de encontrar alternativas de uso y manejo de la especie que generen nuevos sistemas productivos regionales especialmente en la Orinoquia colombiana.

La participación activa de los docentes investigadores en la Orinoquia colombiana juega un papel muy importante, cuando se vincula a los campesinos para 
participar en el proceso investigativo porque de ellos depende gran parte de la elaboración del estudio.

Es necesario establecer contactos en diferentes puntos de la Orinoquia para evaluar el impacto de las actividades de caza y manejo de la especie con el fin de realizar otro tipo de investigaciones.

\section{BIBLIOGRAFÍA}

1. Braga, E.; Camarao, A.P. Effect of forage availability on digestibility of Paspalum plicatulum Mich Vel. Pasturas Tropicales, 9 (2): 24-26. 1987.

2. Drogoul, C.; Poncet, C.; Tisserand, J. L. Feeding ground and pelleted hay rather than chopped hay to ponies 1 . Consequences for the in vivo digestibility and rate of passage of digesta. Anim. Feed Sci. Technol., 8: 117-130. 2000.

3. Emmons, H. L. Neotropical Rainforest Mammals. The University of Chicago, Chicago. 1997.

4. FAO. Organización de las Naciones Unidas para la Agricultura y la Alimentación. Manejo de Fauna Silvestre y Desarrollo Rural, Información sobre 7 especies de América Latina y el Caribe. Proyecto FAO/PNUMA. FP-G 1058501, Documento Técnico No 2, Oficina Regional de la FAO para América Latina y el Caribe, Lima. 1985.

5. Forero J., Betancur J.; Cavelier J. Dieta del capibara Hydrochaeris hydrochaeris (Rodentia: Hydrochaeridae) en Caño Limón, Arauca, Colombia. Revista Biología Tropical, 51 (2): 579-590. 2003.

6. González J., E.; Escobar, A.; Parra R., R. Productividad primaria, secundaria, sistemas de producción actuales y potenciales para las sabanas de Venezuela. Boletín Sociedad Venezolana de Ciencias Naturales, 139: 305324. 1981.

7. Minson, D. J. Nutritional differences between tropical and temperate pastures. En Morley, F.H.W., ed. Grazing Animal. Elsevier, Amsterdam. pp. 143-157. 1981.

8. Morales, M. B.; Berroterán, J. L. Producción y crecimiento de Axonopus purpusii en sabanas de los llanos altos venezolanos. Acta Biológica Venezuelica, 13 (1-2): 137- 150. 1991.

9. Muñoz, A., Fariñas, S.; Ceballos, M. Efecto de la fertilización con fósforo y azufre sobre el valor nutritivo de Axonopus compressus y Desmodium canum. Revista Unellez de Ciencia y Tecnología, 4 (1- 2): 61- 69. 1986a.

10. Muñoz, A.; Fariñas, S.; Ceballos, M. Efecto de la fertilización con fósforo y azufre sobre el valor nutritivo de Paspalum plicatulum y algunas leguminosas nativas. Revista Unellez de Ciencia y Tecnología, 4 (1 - 2): 75-80. 1986b. 
11. Nascimento, J. A. L. de,; Freitas, E. A. G. de,; Duarte, C. M. L. Missionary grass (Axonopus $s p$.) on Santa Catarina plateau: forage production, nutritive value and live weight gain under farm conditions. Boletim Tecnico, Empresa Catarinense de Pesquisa Agropecuaria No 52.65 p. 1990.

12. Ojasti, J.; Burgos, S. Density regulation in population of capybara. Acta Zoologica Fennica, 173: 81-83. 1985.

13. Tejos M. R. Efecto del nitrógeno y fósforo sobre la extracción de nutrientes, recuperación de fertilizantes y valor nutritivo de sabanas de Apure, Venezuela. Revista Unellez de Ciencia y Tecnología, 4 (1-2): 42-49. 1986.

14. Tejos M. R.; Arias N., J. F. Valor nutritivo del pasto lambedora (Leersia hexandra Sw.) durante el período seco de la sabana inundable de Apure, Venezuela. Revista Unellez de Ciencia y Tecnología, 6 (1- 2): 41- 44. 1988.

15. Tejos R. Análisis de crecimiento, valor nutritivo, reservas y descomposición de cinco gramíneas de sabanas inundables. Tesis Doctoral. Universidad Central de Venezuela. Facultad de Agronomía. Maracay, Venezuela, 199 p. 1994.

16. Van Severen, N. L.; Carbonell, R. Estudios de digestibilidad sobre la pulpa de café y la hoja de banano. Café. Salvador, 19: 1619-1624. 1949. 